\title{
On Bit-Rate Selection for Opportunistic Routing
}

\author{
Chase Gray Naveen Santhapuri Srihari Nelakuditi \\ Department of Computer Science and Engineering \\ University of South Carolina, Columbia, SC 29201 \\ Email: \{graycm,santhapu,srihari\}@cse.sc.edu
}

\begin{abstract}
Opportunistic routing (OR) schemes, such as ExOR, have been shown to provide significant throughput gains over traditional best-path routing schemes for wireless networks. Though the performance of $O R$ schemes depend on the bitrate, they currently use a fixed rate for transmitting packets. While several schemes have been proposed for selecting bit-rate for unicast transmission to a single receiver, none of them are suitable for broadcast transmission to multiple receivers under OR. This paper attempts to maximize the benefits of OR with dynamic bit-rate selection. We first define a new metric, Expected Anypath Communication Time (ExACT), that captures the time to deliver a packet to destination with a given rate at each hop under OR. We then propose Bit-rate Selection for Opportunistic Routing (BitSOR) algorithm that minimizes ExACT for each pair of nodes in the network. We evaluate the performance of BitSOR using MIT Roofnet trace and demonstrate significant potential improvement with dynamic rate over $O R$ with the best fixed rate.
\end{abstract}

\section{INTRODUCTION}

Multi-hop wireless mesh networks are becoming increasingly popular for last mile connectivity due to their ease of deployment and low cost. Emerging commercial applications of multi-hop mesh networks such as community wireless networks [1] and city-wide broadband Internet access [2], [3] might be coming to more metropolitan areas in the near future [4]. For most of these applications, the majority of the nodes are stationary and plugged in (not running on battery power). Therefore the focus of most routing algorithms for these networks is to maximize network throughput.

Opportunistic routing (OR) schemes that take advantage of the broadcast nature of wireless transmissions have been actively researched over the past few years [5]-[8]. OR works under the assumption that each transmission is most likely overheard by multiple receivers. The number of nodes overhearing a transmission is partly a function of the bitrate at which the data is transmitted by the sender. If the sender transmits at a low bit-rate, then its packet might be heard by some far away nodes due to low SINR (signal to interference and noise ratio) requirements at lower rates. But it would tie up the wireless channel for many of its neighbors for a longer period of time due to the slower transmission rate. Conversely, if the sender transmits at a higher bit-rate, the packet loss probability would be high and the number of potential receivers might be few [9], but the channel would be occupied for less time. Thus, the bit-rate used for transmitting affects the throughput of an OR scheme significantly. Nevertheless, previously proposed OR schemes use fixed bit-rate, and dynamic bit-rate selection for OR is considered an open problem [8].

There are many schemes proposed for selecting bit-rate at the MAC layer [10]-[13]. But all of these schemes are interested only in determining the best rate for a single receiver. They are suitable for traditional routing protocols that forward the packet to a single next-hop along the chosen path. However, they are quite inadequate for OR where packets are broadcast to multiple potential receivers. While the problem of identifying the best rate for delivering a packet to multiple receivers is hard, it is even more so with OR. The ideal transmission rate under OR has to deliver the packet to at least one of the potential next-hop nodes (candidates), preferably to the highest priority candidate (which is "closer" to the destination than all other candidates). Probably due to the challenges involved in addressing this problem, previously proposed OR schemes use fixed rate [5], [8] transmissions. In this paper, we take a step towards designing a practical dynamic rate selection protocol for OR by developing an offline algorithm for selecting the best rate at each node under OR for each destination.

We make the following contributions in this paper towards maximizing the performance of OR for wireless networks with bit-rate selection: 1) We propose a new routing metric Expected Any-path Communication Time (ExACT) that captures the total transmission time needed to deliver a packet from a given node to its destination sending at the specified rate at each hop under OR. 2) We present the Bit-rate Selection for Opportunistic Routing (BitSOR) algorithm. The algorithm selects the ideal bit-rate, the candidates at that bit-rate, and the priority for each candidate for each possible destination so as to minimize the corresponding ExACT. 3) We evaluate the performance of BitSOR using the MIT Roofnet trace [?] and demonstrate significant potential improvement with dynamic rate selection over OR with the best fixed rate, particularly for distant node pairs.

The remainder of this paper is organized as follows. Section II provides an overview of opportunistic routing and existing bit-rate selection algorithms. Our new path metric for determining the best bit-rate for $\mathrm{OR}$ is presented in Section III. We illustrate the need for bit-rate selection for OR in mesh networks and describe BitSOR in Section IV. The MIT Roofnet trace based evaluation results are presented in Section V. We discuss the challenges in designing a practical scheme in Section VI. Finally, Section VII concludes and outlines areas for further work. 


\section{RELATED WORK}

\section{A. Routing Metrics}

The minimum hop count metric used for routing has been shown to not necessarily maximize the throughput of a flow in a wireless network [14]. Expected number of transmissions(ETX) [14] estimates the number of transmissions needed to deliver a unicast packet by measuring the loss rate of broadcast packets between pairs of neighboring nodes. ETX does not accurately reflect the cost of a link when multiple bit-rates are available. A low rate link with a smaller ETX may actually be slower than a high rate link with a larger ETX. Expected Transmission Time (ETT) [15] addresses this limitation of ETX by accounting for bit-rate. Both ETX and ETT assume that a packet will travel along a single path from source to destination. This is not true for opportunistic routing, as a packet may travel along any one of the many potential paths through candidates. [6] presents a new metric, Expected Anypath Transmissions (EAX), that reflects the number of transmissions needed to deliver a packet from a node to its destination under OR. In this work, we extend EAX to take the bit-rate into account to define a new metric, ExACT.

\section{B. Opportunistic Routing}

Multi-hop wireless networks typically use routing techniques similar to those in wired networks [16], [17]. Recently there have been many schemes proposed that take advantage of broadcast transmissions to send information opportunistically. Extremely opportunistic routing (ExOR) is one such routing scheme [5] where, for each destination, a set of next-hop candidates are selected and each of them is assigned a priority according to its closeness to the destination. The highest priority node is chosen as the next-hop for forwarding, after the packet's transmission, among the candidates that received it. Therefore, the utility of OR hinges on inter-candidate communication and candidate selection. ExOR does not take into account the availability of different bit-rates and hence may not select the best possible candidates. MORE [8] extends ExOR by increasing the usefulness of forwarding duplication due to unsuccessful inter-candidate communication. It achieves this by linearly coding packet combinations at each hop, thus attempting to ensure that each packet has new information. Due to this, they are able to mitigate the need for a robust acknowledgement scheme. In [8], the authors state that they were forced to evaluate $\mathrm{OR}$ at a fixed rate due to the unavailability of an appropriate auto-rate mechanism for OR. This paper explores the scope for solving this problem by developing an offline algorithm for bit-rate selection for OR schemes.

\section{Rate Selection}

The original bit-rate selection algorithm was created for the WaveLAN-II 802.11 cards [10] called Auto Rate Fallback (ARF). Adaptive Auto Rate Fallback (AARF) [11] is an extension of ARF where step-up parameter is doubled every time the algorithm tries to increase the bit-rate and the subsequent packet fails. This is helpful when packet failures take up a large amount of transmissions time. The MadWifi device driver for Atheros cards [18] uses the Onoe algorithm which is much less sensitive to individual packet failure than the ARF algorithm, and basically tries to find the highest bit-rate that has less than $50 \%$ loss rate. This algorithm is relatively conservative; once it decides a bit-rate will not work, it will not attempt to step up again until at least 10 seconds have gone by. Receiver Based Auto-Rate (RBAR) [12] chooses the bit-rate based on S/N measurements at the receiver. In CHARM [19] the authors uses a rate selection algorithm based on a moving average of estimated path-loss at the receiver. In both the above schemes, the basic intuition is that channel quality seen by the receiver is what determines whether a packet can be received. Opportunistic Auto-Rate (OAR) [20] takes advantage of higher bit-rates by opportunistically sending multiple back-to-back data packets whenever the channel quality is good but is not appropriate for networks using OR. SampleRate [13] chooses the bit-rate that it predicts will provide the most throughput based on estimates of the expected per-packet transmission time for each bit-rate. We intend to use the general concepts of SampleRate while designing a practical bit-rate selection scheme for OR.

\section{EXPECTED Any-PATH COMmunication Time} (EXACT)

Current bit-rate selection algorithms try to find the most efficient rate for sending with the assumption of a single receiver and are not applicable to OR. Therefore, OR protocols currently use a fixed sending rate. This brings us to the question: How should auto-rate choose the best sending rate in order to maximize the benefits of OR? In this section we present our path metric for routing which will be used by the bit-rate selection algorithm to select the best rate.

To develop our auto-rate algorithm for opportunistic routing, we need a new path metric that does not assume a single path. This is similar to EAX [6], which provides a metric for capturing the cost from source to destination assuming a packet could traverse any possible path. This new metric is termed Expected Any-Path Communication Time(ExACT). We extend on the formula in [6] to account for all possible sending rates. Because nodes could be sending at different rates we must normalize each value to total communication time instead of total number of transmissions, similar to [15]. Due to the 802.11 physical layer preamble being transmitted at the lowest possible rate, we must take this into account when determining the "effective" bit-rate. In this paper we are focusing our work with $802.11 \mathrm{~b}$, which has a preamble time of $192 \mu \mathrm{s}$. Due to this, the "effective" bit-rate is closer to optimal as the packet sizes increase because the preamble time becomes a smaller portion of total communication time. The values calculated for $802.11 \mathrm{~b}$ can be seen in Table I.

To simplify computation, we assume that acknowledgement reception ratio is $100 \%$. This is a reasonable assumption if an opportunistic routing scheme such as MORE [8] is used that does not require strict coordination among candidate nodes for forwarding. We calculate the total communication time to transmit a packet from a source $s$ to a destination $d$, given 


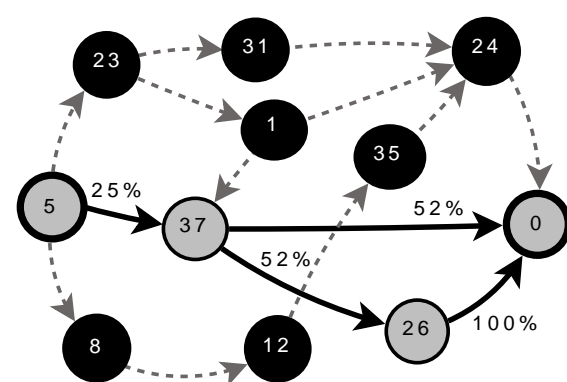

(a) $5.5 \mathrm{Mbps}$

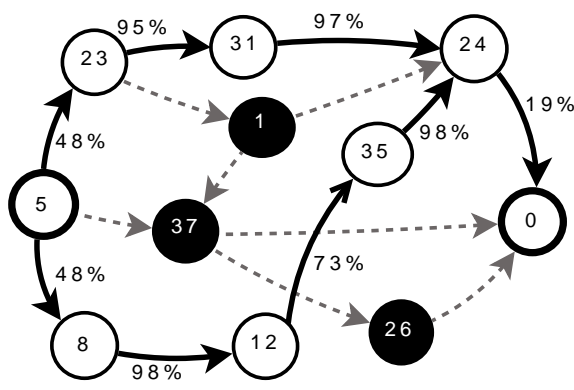

(b) $11 \mathrm{Mbps}$

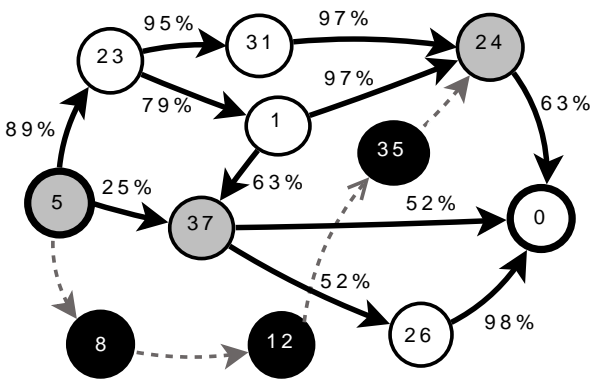

(c) Dynamic Rate

Fig. 1. Sample topologies, extracted from Roofnet trace, at $5.5 \mathrm{mbps}, 11 \mathrm{mbps}$ and optimal opportunistic rate for source node 5 and destination node 0. White nodes indicate node using $11 \mathrm{mbps}$, gray nodes indicates node using $5.5 \mathrm{mbps}$ for broadcast, black nodes indicate unused nodes for that network instance. Invalid or unused links are represented with gray dotted arrows and valid links are represented with solid black arrows.

TABLE I

EFFECTIVE SENDING RATES FOR 802.11B

\begin{tabular}{c||c|c||c|c} 
Bit-rate & 200 bits & 1000 bits & 4000 bits & 12000 bits \\
\hline 1 & 0.5102 & 0.83893 & 0.9542 & 0.98425 \\
2 & 0.68493 & 1.44509 & 1.82482 & 1.93798 \\
5.5 & 0.8758 & 2.6751 & 4.35127 & 5.05515 \\
11 & 0.95156 & 3.5347 & 7.19895 & 9.35374
\end{tabular}

the candidate set $C^{s, d}$. Suppose candidate $c_{i}$ is the candidate with priority $i$ (with 1 being the highest). Suppose that packet delivery probability from $s$ to $C_{i}^{s, d}$ is $f_{i}$. Each node has a set of available rates. Let $r$ denotes a specific effective sending rate (in Mbps) at node $s$, and $r_{i}^{*}$ denote the ideal transmission rate for packets from $c_{i}$ to $d$. Then we have,

$\operatorname{ExACT}(s, d, r)=\frac{\frac{1}{r}+\sum_{i} \operatorname{ExACT}\left(c_{i}, d, r_{i}^{*}\right) f_{i} \prod_{j=1}^{i-1}\left(1-f_{j}\right)}{1-\prod_{i}\left(1-f_{i}\right)}$

The above equation is composed of three core parts. $1 / r$ is the amount of time it takes to transmit a single packet at bitrate $r . \operatorname{ExACT}\left(c_{i}, d, r_{i}^{*}\right) f_{i} \prod_{j=1}^{i-1}\left(1-f_{j}\right)$ is time from $c_{i}$ to $d$ taking into account the probability that $s$ delivers successfully to $c_{i}$ and fails to deliver to higher priority candidates. Finally we divide by $1-\prod_{i}\left(1-f_{i}\right)$, which is the probability of successfully delivering the packet to one of the candidates. The candidate selection process is explained in much more depth in [?].

\section{Bit-RATE SELECtion FOR OpPortunistic Routing (BITSOR)}

The bit-rate that yields the minimum ExACT value is the ideal rate at which source node $s$ should broadcast a packet to its candidates corresponding to destination $d$. Since the ExACT expression is recursive, the computation starts at the destination and works backwards, calculating the minimum ExACT value at each candidate next-hop until the minimum ExACT value and the corresponding rate $r^{*}$ at the source is known. This resulting value is the total time to transmit a single packet at rate $r^{*}$ from $s$ to $d$ with the candidate set $C^{s, d}$. We call our rate selection algorithm BitSOR (Bit-rate Selection for Opportunistic Routing).

\section{A. Illustration}

Fig. 1 lists a set of topologies relevant for opportunistic routing between source node 5 and destination node 0 . These were extracted from the Roofnet trace for two different bitrates, 5.5 Mbps in Fig. 1a and $11 \mathrm{Mbps}$ in Fig. 1b. Fig. 1c is the resulting network when each node determines the best sending rate using the ExACT metric. The ExACT values can be seen in Table II, ??, and IV. We include all nodes used at any of the rates chosen. This is to help illustrate the changing opportunities at each node when selecting the best sending rate. Unused nodes in a certain network instance are shaded black, and their unused links are dashed and shaded gray. The solid black links show connections to each candidate that makes forward progress based on the ExACT metric for destination node 0 .

For illustration, let's look at node 24 in Fig. 1. In Fig. 1a it is not involved in the forwarding at all. The ExACT values for nodes 23 and 8 do not indicate forward progress with a fixed network rate of 5.5 Mbps, so we do not have a path traveling through node 24. In Fig. 1b, node 24 is involved in forwarding and it has chosen a rate of $11 \mathrm{Mbps}$. Node 24 is actually the only node with a connection to destination node 0 . Finally, in Fig. 1c with dynamic rate selection, node 24 has been chosen to be one among the three nodes to send at $5.5 \mathrm{Mbps}$. It is also no longer the bottleneck node along the path to destination node 0 . Source node 5 has gained many more chances for opportunistic routing and its resulting ExACT value reflects this. By using rate selection for our opportunistic routing, we have gained more routes and have also been able to avoid a possible bottleneck link from node 24 to node 0 . These types of gains occur frequently in the Roofnet trace data. We explore the results in more detail in Section V.

\section{B. Routing Protocol}

Our opportunistic routing protocol is based on the proposed ExACT metric. It involves the updating of ExACT values, selecting candidates, and prioritizing them. After the ExACT values are calculated for each node and each rate, the candidate set is constructed. All neighboring nodes that have a lower ExACT value to the destination than the current 
node (in other words that make forward progress to the destination) are included in the set. The candidates are then prioritized based on the ExACT values (least value candidate has highest priority). Gathering the delivery probabilities for ExACT values can be done by periodic probes [13] or by estimating reception probability at the receiver like in [19]. If there is bidirectional traffic or ACKs, this information can be continually updated if the receiver embeds the delivery probability in the reverse traffic. The interval between ExACT value updates can be selected based on the frequency of delivery probability changes. In [13] it was shown that any updates within 10 seconds are not helpful but this can vary from one topology to another. We are currently working on a practical opportunistic routing protocol taking these aspects into consideration.

TABLE II

VALUES FOR FIG. 1A

\begin{tabular}{c||c|c} 
Node & ExACT & Cands \\
\hline 5 & 13348 & 37 \\
37 & 3854 & 26,0 \\
26 & 2373 & 0 \\
& & \\
& &
\end{tabular}

TABLE III VALUES FOR FIG. 1B

\begin{tabular}{c||c|c} 
Node & ExACT & Cands. \\
\hline 5 & 11650 & 23,8 \\
23 & 9290 & 31 \\
8 & 11000 & 12 \\
31 & 7940 & 24 \\
12 & 9690 & 35 \\
35 & 7930 & 24 \\
24 & 6620 & 0
\end{tabular}

\section{Evaluation}

In this section we present offline simulation results to assess the gains that can be obtained by using an optimal bit-rate selection algorithm for OR. We used the publicly available Roofnet trace data, obtained from 90 second transmissions by each node in the network. The first set of results are based on the average delivery ratios over the entire time for each link. For the second set of results, we extracted the delivery ratios for each link during 85 one-second snapshots of the network.

\section{A. Topology based on delivery ratios over entire trace}

Our first evaluation is the comparison of total time for dynamic rate vs. fixed rate opportunistic schemes for each (src,dest) pair. Fig. 2 shows scatter plots of all node pairs for $11 \mathrm{Mbps}$ and 5.5 Mbps respectively. In the $11 \mathrm{Mbps}$ case, most of the node pairs perform only slightly better with dynamic rate but for some node pairs fixed rate can be four times worse. In contrast, 5.5 Mbps performs consistently worse than 11 Mbps but the most variation seen is less than half. This would imply that always sending at $11 \mathrm{Mbps}$ would result in more

TABLE IV

VALUES FOR FIG. 1C

\begin{tabular}{c||c|c|c|c|} 
Node & ExACT(in $\mu$ s) & Rate (mbps) & Improvement & Cands \\
\hline 5 & 8080 & 5.5 & $30.6 \%$ & 37,23 \\
37 & 3510 & 5.5 & N/A & 0,26 \\
23 & 6240 & 11 & $32.8 \%$ & 1,31 \\
1 & 4910 & 11 & N/A & 37,24 \\
31 & 5100 & 11 & $35.7 \%$ & 24 \\
26 & 1310 & 11 & N/A & 0 \\
24 & 3780 & 5.5 & $42.9 \%$ & 0
\end{tabular}

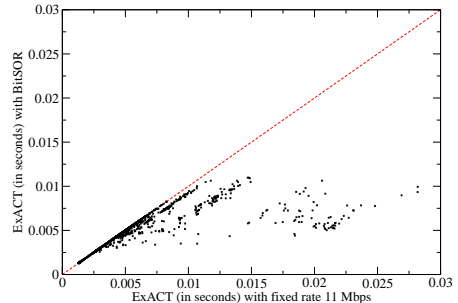

(a) $11 \mathrm{Mbps}$

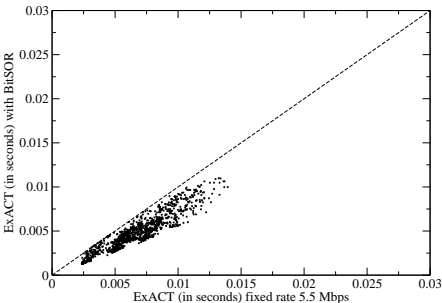

(b) $5.5 \mathrm{Mbps}$
Fig. 2. The ExACT value (in seconds) with opportunistic routing with dynamic rate vs. opportunistic routing with various fixed rates. The packet size is 12000 bits.

frequent path cost changes that might have to be updated at neighboring nodes. Conversely, 5.5 Mbps is much less prone to have constantly varying ExACT values.

The box plot in Fig. 3 shows the mean, median and standard deviation for the ExACT values of dynamic rate and all fixed rates. The error bars show the first and fourth quartile to the minimum and maximum ExACT values respectively. We can again see that the variation of ExACT values at $11 \mathrm{Mbps}$ is greater than the average dynamic rate ExACT value for the entire fourth quartile. Fig. 4 shows the gain over fixed-rate schemes for a different number of hops. The gains are higher for four and five hops and these results also corroborate the results in Fig. 2 and 3 showing a number of pairs performing very well with optimal dynamic rate while many perform about equal. The number of (src,dest) pairs selecting a data rate varies with the size of the packet and is shown in Fig. 5. At lower packet sizes there are more 5.5 Mbps links but as the packet size increases, the $11 \mathrm{Mbps}$ links dominate the network. This is because of the physical preamble overhead $(192 \mu \mathrm{s})$ whose significance decreases with an increase in packet size. This results in a higher increase in effective rate of $11 \mathrm{Mbps}$ at higher packet size when compared with 5.5 Mbps and thereby reduces the ExACT value for $11 \mathrm{mbps}$ links.

\section{B. Snapshot topologies based on one second averages}

Signal conditions vary frequently and the resulting changes to delivery ratios and the topology may effect the optimal bit-rate. To see this affect we created topology snapshots by extracting delivery ratios of all links for each second. By measuring the improvement variations and optimal-rate variations we show how often the optimal rates change and how many (src,dest) pairs operate at a given optimal rate. Fig. 6 and Fig. 7 show the aggregate ExACT improvement over fixed rates for each snapshot for 12000-bit packets and 4000bit packets respectively. It can be observed that at lower packet sizes there are more links using 5.5Mbps (resulting in a lower speedup value) and hence the resulting gain from fixed rate $5.5 \mathrm{Mbps}$ reduces while the gain over $11 \mathrm{Mbps}$ increases. We can also see again the stability of $5.5 \mathrm{Mbps}$ even over 85 one second intervals. In Fig. 8, the number of times the selected rate changes over the entire trace time at one second intervals is shown for each (src,dest) pair. We can see that there is a significant amount of change occurring for each node pair. 


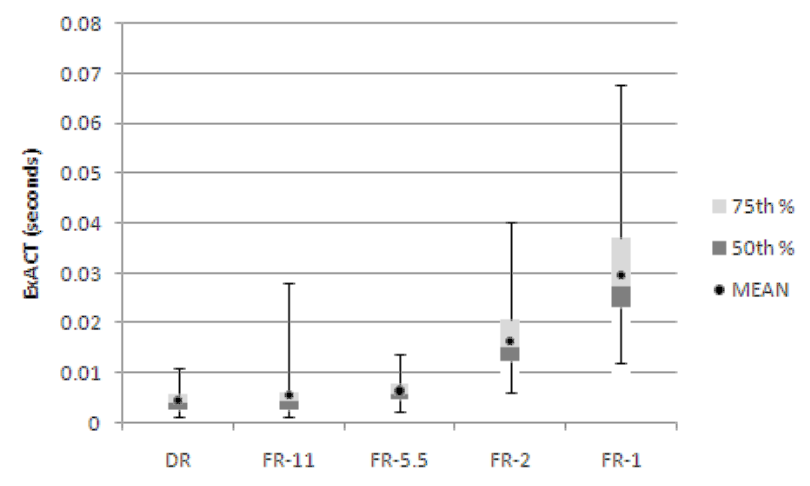

Fig. 3. Comparisons between ExACT values for opportunistic routing with dynamic rate (OR-DR) vs. opportunistic routing with fixed rate (OR-FR). The packet size is 12000 bits. (a) $11 \mathrm{mbps}$

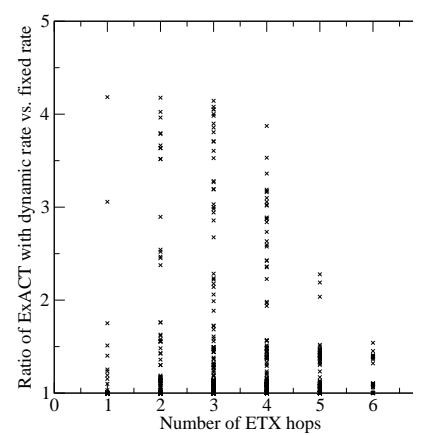

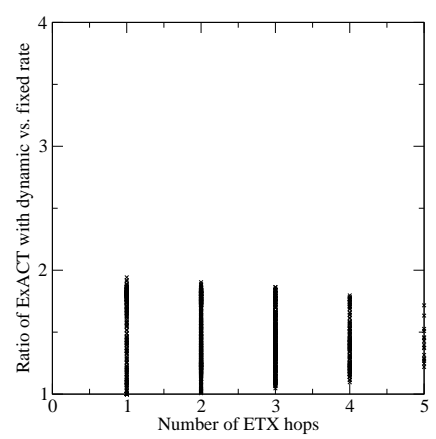

(b) $5.5 \mathrm{mbps}$
Fig. 4. The gain obtained as number of hops increase. OR-DR vs. OR-FR with a packet size of 12000 bits.

Overall we have found significant improvements can be found by selecting appropriate rates in opportunistic routing. Some of the potential is not reflected in the Roofnet topologies because of the fewer (4) $802.11 \mathrm{~b}$ rates and fewer nodes in the network. It is also due to the delivery ratios measured without any internal interference which enables a disproportionately large number of $11 \mathrm{Mbps}$ links as shown in Fig. 9. With a mesh network using 802.11a radios, there are a total of 8 data rates available and the need for selecting optimal rate might be even more significant. We plan to test this hypothesis on a testbed soon.

\section{Challenges And Discussion}

Bit-rate selection for OR can be approached in many ways. Simple local heuristics can be used to reduce state propagation overhead but might not give a global improvement. Two intuitive ideas can be picking the rate which is best for most of the candidates and picking the best-rate of the next-hop candidate with the best ETT. It is not immediately clear if these approaches will result in a near-optimum value. BitSOR selects rates that minimize the ExACT values.

Thus far we have presented an offline bit-rate selection protocol which requires each node to have the delivery ratio information from every other node in the network. This is

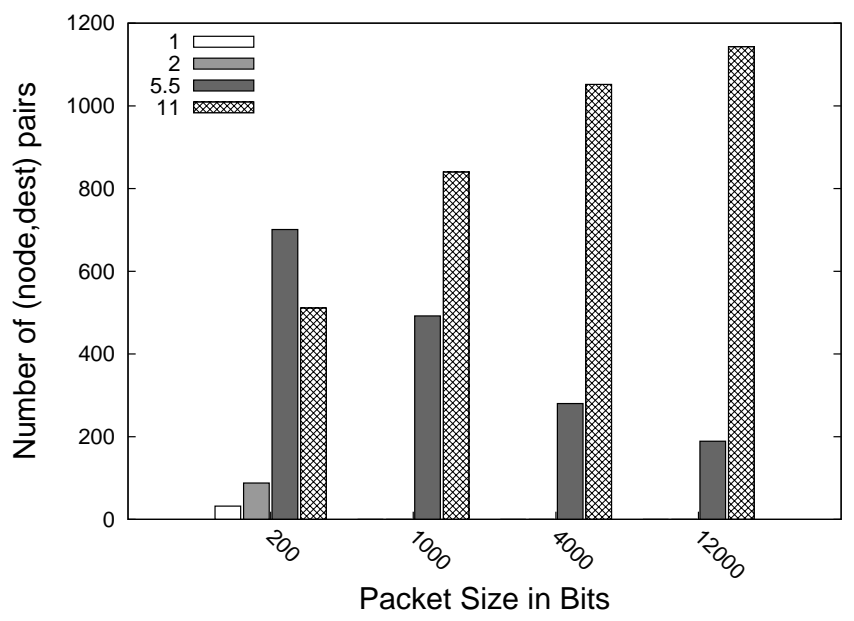

Fig. 5. Actual bit rates selected for each source destination pair at each nacket size

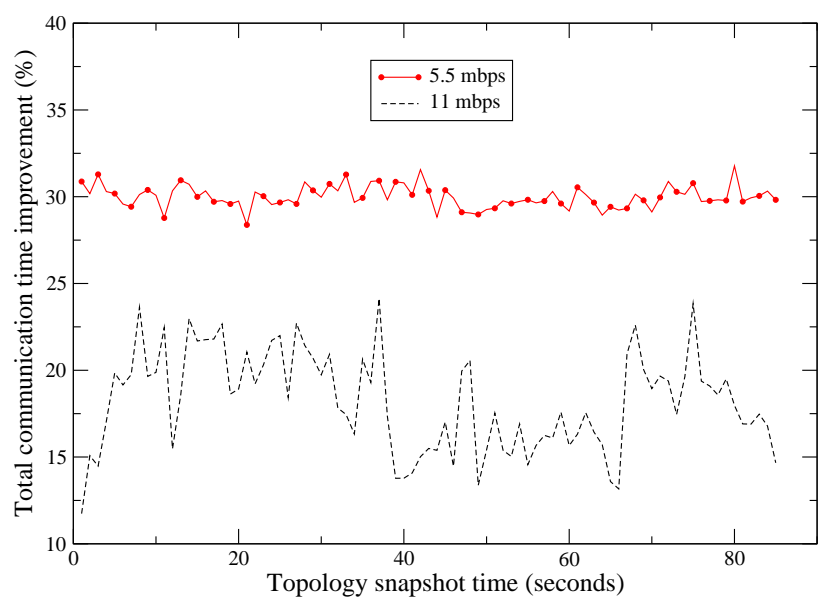

Fig. 6. Percentage improvement in total communication time for 85 one second tonoloov snanshots 1 T) no hit nackets

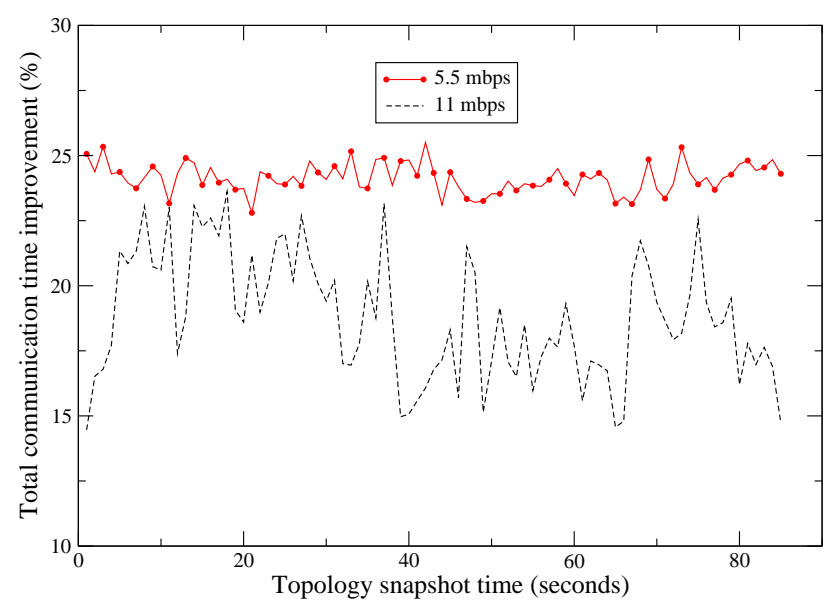

Fig. 7. Percentage improvement in total communication time for 85 one second topology snapshots. 4000 bit packets 

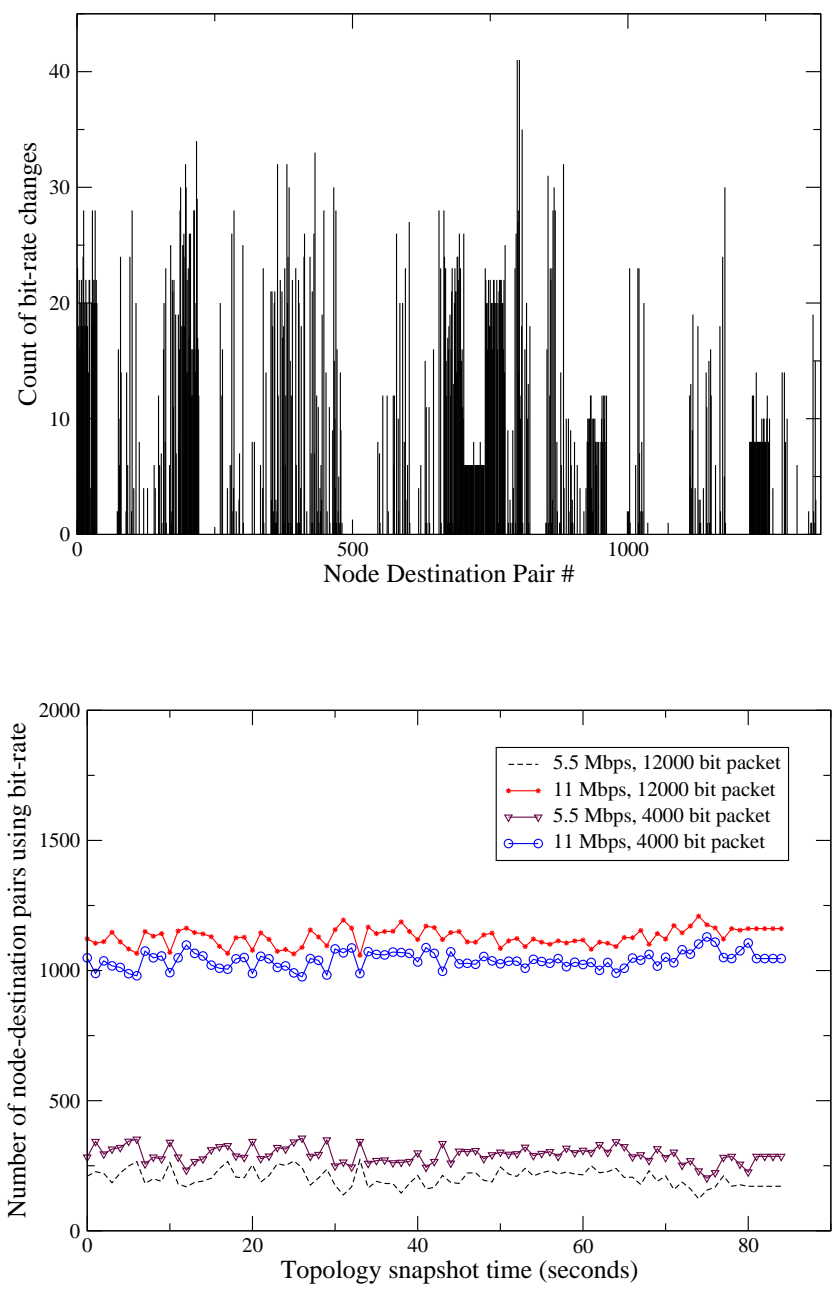

Fig. 9. Total node-destination pairs using a certain rate for each one second topology snapshot.

not practical for a real-time rate selection algorithm. We are working on a hybrid distance vector and link state routing protocol. Using this approach, nodes periodically flood their best estimate ExACT values to each destination. This information is used to maintain relative consistency among nodes. Between link state updates, nodes piggyback updated ExACT information on opportunistic packets. Because nodes interested in using this forwarder should most likely overhear when the forwarder transmits the packet, updated ExACT information would trickle in the reverse path direction beginning at neighbors of the destination. Nodes that cannot overhear these piggybacked updates would not be at a loss because the forwarder is probably out of range or has a low delivery ratio.

To reduce the effect of frequently changing ExACT values in the network, nodes do not recalculate them unless the updated ExACT value is significantly differing from the current value within a threshold. Furthermore, nodes may prefer to maintain consistent ExACT values as opposed to minimizing them. From our results, a node could maintain a more consistent ExACT value by using 5.5 Mbps. By using this lower rate, ExACT time is not significantly lower, but consistency is much higher and distance vector updates would be much less frequent. If the node wished to choose $11 \mathrm{Mbps}$, it may choose to not update when its ExACT value varies but, on average converges to its reported ExACT value.

\section{CONCLUSION AND FUTURE WORK}

Bit-rate selection for opportunistic routing is an interesting open problem. In this work we address this problem by proposing a new metric, ExACT, and a new bit-rate selection algorithm, BitSOR, to select the ideal rate for OR. Our evaluation based on the Roofnet trace data has shown that dynamic rate selection performs significantly better than OR with the best fixed rate, particularly for distant node pairs. It also indicates the scope for further improvement in other topologies with $802.11 \mathrm{~b}$ and networks using 802.11a radios. We are currently running the MORE [8] implementation on our testbed of Atheros chipset based routers, which we are extending to use BitSOR to determine the appropriate sending rate.

\section{REFERENCES}

[1] B. A. Chambers, "The Grid Roofnet: A Rooftop ad hoc wireless network," MS Thesis, MIT, 2002.

[2] "Seattle wireless," http://www.seattlewireless.net.

[3] "Bay area wireless users group," http://www.bawug.org.

[4] "Austin wireless," http://www.austinwirelesscity.org.

[5] S. Biswas and R. Morris, "Opportunistic routing in multi-hop wireless networks," in ACM SIGCOMM, Aug. 2005.

[6] Z. Zhong and S. Nelakuditi, "On the efficacy of opportunistic routing," in Proceedings of SECON'07. IEEE, 2007.

[7] R. C. Shah, S. Wietholter, A. Wolisz, and J. M. Rabaey, "When does opportunistic routing make sense?" in PERCOMW '05: Proceedings of the Third IEEE International Conference on Pervasive Computing and Communications Workshops. Washington, DC, USA: IEEE Computer Society, 2005, pp. 350-356.

[8] S. Chachulski, M. Jennings, S. Katti, and D. Katabi, "Trading structure for randomness in wireless opportunistic routing," in ACM SIGCOMM New York, NY, USA: ACM, 2007, pp. 169-180.

[9] D. Aguayo, J.Bicket, S.Biswas, G.Judd, and R. Morris, "Link-level measurements from an 802.11 b mesh network," in ACM SIGCOMM 2004, Aug. 2004.

[10] A. Kamerman and L. Monteban, "Wavelan(c)-ii: a high-performance wireless lan for the unlicensed band," Bell Labs Technical Journal, vol. 2, no. 3, pp. 118-133, 1997, http://dx.doi.org/10.1002/bltj.2069.

[11] M. Lacage, M. H. Manshaei, and T. Turletti, "Ieee 802.11 rate adaptation: A practical approach," citeseer.ist.psu.edu/719742.html.

[12] G. Holland, N. H. Vaidya, and P. Bahl, "A rate-adaptive MAC protocol for multi-hop wireless networks," in Mobile Computing and Networking, 2001, pp. 236-251, citeseer.ist.psu.edu/holland01rateadaptive.html.

[13] J. C. Bicket, "Bit-rate selection in wireless networks," 2005 , http://dspace.mit.edu/handle/1721.1/34116.

[14] D. D. Couto, D. Aguayo, J. Bicket, and R. Morris, "A high-throughput path metric for multi-hop wireless routing," in Proc. ACM Mobicom, 2003.

[15] R. Draves, J. Padhye, and B. Zill, "Routing in multi-radio, multi-hop wireless mesh networks," in Proc. ACM Mobicom, 2004.

[16] C. Perkins, "Ad-hoc on-demand distance vector routing," 1997, citeseer.ist.psu.edu/article/perkins97adhoc.html.

[17] C. Perkins and P. Bhagwat, "Highly dynamic destination-sequenced distance-vector routing (DSDV) for mobile computers," in ACM SIG COMM, 1994, pp. 234-244, citeseer.ist.psu.edu/perkins94highly.html.

[18] "Madwifi," http://sourceforge.net/projects/madwifi.

[19] G.Judd, X. Wang, and P. Steenkiste, "Extended abstract: Lowoverhead channelaware rate adaptation," in Proc. ACM Mobicom, 2007.

[20] B. Sadeghi, V. Kanodia, A. Sabharwal, and E. Knightly, "OAR: an opportunistic auto-rate media access protocol for ad hoc networks," Wirel. Netw., vol. 11, no. 1-2, pp. 39-53, 2005. 